\title{
Preserving emittance by matching out and matching in plasma wakefield acceleration stage
}

\author{
Xiangkun Li, Antoine Chancé, and Phu Anh Phi Nghiem* \\ CEA-Irfu, Centre de Saclay, Université Paris-Saclay, 91191 Gif sur Yvette, France
}

(Received 28 August 2018; published 21 February 2019)

\begin{abstract}
In more than four decades, particle acceleration by plasma wakefield has demonstrated its feasibility and efficiency. This acceleration technique is now starting to be planned for providing high-quality beams to well-defined user communities. High beam energy is also considered by piling successive plasma acceleration stages. In this context, avoiding beam degradation, on top of all emittance degradation, is the main concern when transferring the accelerated beam to the users or to the following acceleration stage. After examining the behavior of the trace and the phase emittances when crossing through a conventional transfer line, we are able to determine the criteria to be achieved in the plasma ramps so as to minimize emittance growth. Then the optimal density profile is studied for these ramps at the entrance and exit of a plasma stage accelerating electrons from the energy of $150 \mathrm{MeV}$ to $5 \mathrm{GeV}$. Finally, the design of an optimal transfer line allows showing that the emittance growth can be contained to less than $10 \%$ in realistic conditions when transferring a beam to a free-electron laser.
\end{abstract}

DOI: 10.1103/PhysRevAccelBeams.22.021304

\section{INTRODUCTION}

From the suggestion to use a plasma wakefield to accelerate charged particles [1], the proof of this principle is now well established theoretically and experimentally $[2,3]$. Induced by either a particle beam delivered by a conventional accelerator [4] or a photon beam provided by a laser [5], huge wakefields in plasma columns have been observed, accelerating electron beams to the multi-GeV energy range. This acceleration technique has reached nowadays its maturity. Its feasibility and its efficiency are no more to be proven. Laser-plasma facilities are being used as $\mathrm{x}$-ray photon sources [6,7], and plasma wakefield projects aiming at providing routinely a high-quality beam to welldefined user communities have started to be planned [8-10]. For the free-electron laser community, the beam quality in terms of emittance and energy spread is primordial. For the high-energy physics community, the increase to higher and higher energy is essential, which is possible by stacking successive acceleration stages $[11,12]$. In all cases, beam extraction from the plasma stage for transferring either to the users or to the next plasma stage is a key point.

This extraction and transfer, if done without carefully matching the beam, can dramatically damage its quality

\footnotetext{
*Corresponding author. phu-anh-phi.nghiem@cea.fr

Published by the American Physical Society under the terms of the Creative Commons Attribution 4.0 International license. Further distribution of this work must maintain attribution to the author(s) and the published article's title, journal citation, and DOI.
}

characterized by its emittance. As pointed out in Ref. [13], a mismatched beam will induce envelope oscillations leading to significant emittance growth after betatron decoherence. The electric wakefield being huge, its longitudinal component is very efficient for particle acceleration, and the same goes for its transverse gradient with respect to particle focusing. Compared to focusing elements in a conventional transfer line, quadrupoles or solenoids, the focusing gradient $\mathrm{K}\left(\mathrm{m}^{-2}\right)$ of the wakefield can be 2 or 3 orders of magnitude stronger. When passing abruptly from a given focusing region to another completely different, an important mismatch is induced, causing a big jump in emittance. In Ref. [14], an adiabatic ramp of $\mathrm{K}$ has been derived for the transition zone between the two different focusing regimes in order to avoid emittance growth, and in Ref. [15] it is shown that a linear variation of the laser beam size allows one to achieve the indicated adiabatic profile. Almost no emittance growth can be obtained in this way for a weak charge, in the absence of beam loading effects and at the condition to adjust a parameter governing the ramp length.

A facility aiming at delivering a beam routinely to a user community often requires, in addition to high beam quality, a high beam charge and a high reliability. It is therefore important to study the behavior of beam emittance in the presence of a beam loading effect and to check the efficiency of plasma ramps for mitigating emittance growth that can be achieved easily like a simple density ramp.

Besides, the issue of emittance growth is very often analyzed only for the phase emittance in a free drift. Yet transfer lines also include focusing elements, and the trace emittance is more meaningful, as all rms beam parameters can be deduced from it. It is therefore useful to clarify the 
three following points: (a) Which emittance, phase or trace emittance, should be preserved? (b) In which context, free drift or focusing element, does emittance growth occur? (c) Which parameters govern the emittance growth? Answering thoroughly and consistently those three questions allows one to know how to mitigate emittance growth.

In this paper, we will start to derive the expressions governing the evolution of trace emittance and phase emittance in the transfer line downstream from the plasma exit, for both free drifts and focusing elements. From that, the exact relation between the two emittances can be deduced, and all the parameters involved in their evolution can be precisely identified. This allows us to highlight the criteria minimizing emittance growth and the role of different components that should contribute to this minimization. Those analytical expressions are thereafter confirmed by particle tracking simulations. Then we examine the case of a plasma stage accelerating a $30 \mathrm{pC}$ electron beam at $150 \mathrm{MeV}$, ending up at $5 \mathrm{GeV}$ with a small energy spread of $1 \%$ and a small normalized emittance of $1 \mathrm{~mm}$ mrad. Linear and exponential density down ramps are simulated and their length adjusted so as to minimize emittance growth. The results are compared to those obtained with the cited adiabatic ramp of K. Density up ramps at the plasma entrance are also studied to check their ability to ease beam injection as well. Finally, a transfer line including permanent and electromagnetic quadrupoles is designed and optimized, driving the beam from the plasma down ramp to a free-electron laser (FEL). Thanks to that, the beam emittance delivered at the user door can be mitigated and estimated in more realistic conditions.

\section{EMITTANCE EVOLUTION}

A particle distribution being given, the motion of each particle in the $(x, z)$ space, with $x$ and $z$ its transverse and longitudinal positions, respectively, can be fully described with the additional knowledge of the corresponding momenta $\left(p_{x}, p_{z}\right)$, the conjugate variables in Hamiltonian dynamics. Instead of the momentum $p_{x}$, the slope $x^{\prime}$ can also be considered. It is defined by

$$
x^{\prime}=\frac{p_{x}}{p_{z}} .
$$

The emittance is the measure of the surface of the ellipse representing (in the rms meaning) the distribution of particles in the transverse space, which is either $\left(x, x^{\prime}\right)$ or $\left(x, p_{x}\right)$. From these two possible coordinate sets, two kinds of emittance can be defined, the trace emittance and the phase emittance, with the corresponding normalized emittances so as to get rid of their variations with beam energy.

\section{A. Trace emittance and phase emittance}

The 2D emittance is the square root of the determinant of the covariance matrix. The trace emittance and the normalized trace emittance are thus defined, respectively, as

$$
\varepsilon_{\mathrm{tr}}=\sqrt{\left\langle x^{2}\right\rangle\left\langle x^{\prime 2}\right\rangle-\left\langle x x^{\prime}\right\rangle^{2}}
$$

and

$$
\varepsilon_{\mathrm{tr}, n}=\beta_{r} \gamma_{r} \varepsilon_{\mathrm{tr}},
$$

where $\beta_{r}$ and $\gamma_{r}$ are the relativistic coefficients (not to be confused with the Twiss parameters below).

The rms beam size, beam divergence, and correlation are given, respectively, by

$$
\begin{aligned}
& \sigma_{x}^{2}=\left\langle x^{2}\right\rangle=\beta \varepsilon_{\mathrm{tr}}, \\
& \sigma_{x^{\prime}}^{2}=\left\langle x^{\prime 2}\right\rangle=\gamma \varepsilon_{\mathrm{tr}},
\end{aligned}
$$

and

$$
\sigma_{x x^{\prime}}=\left\langle x x^{\prime}\right\rangle=-\alpha \varepsilon_{\mathrm{tr}},
$$

where $\alpha, \beta, \gamma$ are the Twiss parameters. According to Eq. (2), they are linked by the relation

$$
\beta \gamma-\alpha^{2}=1
$$

On the other side, the phase emittance and the normalized phase emittance are defined, respectively, as

$$
\varepsilon_{\mathrm{ph}}=\sqrt{\left\langle x^{2}\right\rangle\left\langle p_{x}^{2}\right\rangle-\left\langle x p_{x}\right\rangle^{2}}
$$

and

$$
\varepsilon_{\mathrm{ph}, n}=\frac{\epsilon_{\mathrm{ph}}}{m_{0} c}
$$

with $m_{0}$ the electron rest mass and $c$ the speed of light.

Assuming that the transverse distributions are centered and that longitudinal and transverse distributions are independent, which is generally the case, the detailed calculations in the Appendix A lead to the relation between the two emittances:

$$
\varepsilon_{\mathrm{ph}, n}^{2}=\varepsilon_{\mathrm{tr}, n}^{2}\left(\frac{\overline{p_{z}^{2}}}{p_{0}^{2}}+\alpha^{2} \frac{\sigma_{p}^{2}}{p_{0}^{2}}\right),
$$

calling $p_{0}$ the average of the $p_{z}$ distribution, which is given by

$$
p_{0}=m_{0} c \beta_{r} \gamma_{r} .
$$

This implies that, when $\alpha=0$, i.e., at a beam waist, where the beam changes from divergent to convergent and vice versa, the two normalized emittances are equal:

$$
\varepsilon_{\mathrm{ph}, n}=\varepsilon_{\mathrm{tr}, n} \quad \text { when } \alpha=0 .
$$


This is often the case at a focusing element, and it is recommended to perform the emittance measurement immediately after those locations [14] in order to get relevant results. In the general case, $\varepsilon_{\mathrm{ph}, n}^{2}$ and $\varepsilon_{\mathrm{tr}, n}^{2}$ differ by the term $\alpha^{2} \sigma_{p}^{2}$, meaning that the two emittances are even more different from each other for a higher energy spread and when the beam is more divergent or else convergent.

As discussed in the Appendix A, Eq. (10) is no longer valid in the presence of strong focusing elements which introduce significant dependences between $x^{\prime}$ and $p_{z}$, but the property given by Eq. (12) remains always true. More generally, Eqs. (10) and (12) show that phase and trace emittances are linked, and thus the growth of both should be mitigated.

Let us study now the evolution of the two emittances in a transfer line. The latter being a combination of drifts and focusing elements, their behavior can be completely described when examining the transfer through a free drift where there is no slope change and that through a thin lens where there is no position change. This will be done in the following, and the derivation of emittance change through a general transfer line is given afterwards.

\section{B. Emittance evolution through a free drift}

Detailed calculations in the Appendix B demonstrate that, when transverse and longitudinal distributions are independent, through a drift of length $l$, the trace emittance remains constant, whereas the phase emittance varies:

$$
\begin{gathered}
\varepsilon_{\mathrm{tr}}^{2}-\varepsilon_{\mathrm{tr} 0}^{2}=0 ; \\
\varepsilon_{\mathrm{ph}, n}^{2}-\varepsilon_{\mathrm{ph} 0, n}^{2}=\varepsilon_{\mathrm{tr} 0, n}^{2}\left(\frac{\sigma_{p}}{p_{0}}\right)^{2} \gamma_{0} l\left(\gamma_{0} l-2 \alpha_{0}\right),
\end{gathered}
$$

where the subscript 0 corresponds to the drift entrance and no subscript corresponds to the drift exit.

This points out that the variation of phase emittance in a drift is higher when the initial trace emittance or the energy spread or the Twiss parameter $\gamma_{0}$ is bigger. It is, for example, noted in Ref. [16] that emittance grows more with a bigger beam emittance and divergence. Those parameters are known to be particularly big in wakefield acceleration, such that in Refs. $[17,18]$, for example, it is concluded that very important emittance growth is unavoidable when transferring the accelerated beam to a user. However, when decomposing the beam divergence in factors of emittance and $\gamma_{0}$ separately as in Eq. (14), we are able to suggest to use the plasma down ramp for minimizing $\gamma_{0}$ (the Twiss parameter at the drift entrance) in order to minimize phase emittance growth, while $\varepsilon_{\text {tr0 }}^{2}$ and $\sigma_{p}^{2}$ are determined in the acceleration process. Indeed, $\gamma_{0}$ being constant through a free drift and very big in the accelerating plasma where wakefield focusing forces are huge, it can only be lowered in the down ramp where these forces progressively and quickly decrease to zero. On the contrary, if $\gamma_{0}$ is not minimized at this specific location, no action in the plasma plateau or in the transfer line can avoid an emittance explosion right at the plasma exit. In short, dissociating the emittance and $\gamma_{0}$ allows pointing out the different mechanisms they are submitted to, finding out the only location where $\gamma_{0}$ can be reduced, and therefore proposing a new way to mitigate emittance growth.

The phase emittance also increases with the drift length, which should thus be the shortest possible so as to limit emittance growth [19]. Equation (14) allows us to calculate the chromatic length $L_{c}$ [20], the length over which the phase emittance increases by a factor of $\sqrt{2}$ when starting from a waist. When $\alpha_{0}=0$,

$$
\frac{\varepsilon_{\mathrm{ph}}^{2}-\varepsilon_{\mathrm{ph} 0}^{2}}{\varepsilon_{\mathrm{ph} 0}^{2}}=\frac{\sigma_{x_{0}^{\prime}}^{2}}{\sigma_{x_{0}}^{2}} \frac{\sigma_{p}^{2}}{\overline{p_{z}^{2}}} l^{2}=\gamma_{0}^{2} l^{2} \frac{\sigma_{p}^{2}}{\overline{p_{z}^{2}}},
$$

and $L_{c}$ is obtained by equalizing the above ratio to 1 and taking the approximation of Eq. (A12):

$$
L_{c}=\frac{\sigma_{x_{0}}}{\sigma_{x_{0}^{\prime}} \frac{\sigma_{p}}{p_{0}}}=\frac{1}{\gamma_{0} \frac{\sigma_{p}}{p_{0}}} .
$$

Here also, it is clear that minimizing $\gamma_{0}$ with the plasma down ramp is the solution allowing one to increase the chromatic length and thus to limit emittance growth. $\gamma_{0}$ is really the parameter that should be taken into consideration, which is furthermore a constant parameter through a free drift.

\section{Emittance evolution through a thin lens}

Through a thin lens of integrated normalized gradient $k=K L$, the derivations developed in the Appendix $\mathrm{C}$ show that, unlike the free drift case, the trace emittance varies and the phase emittance remains constant:

$$
\begin{gathered}
\frac{\varepsilon_{\mathrm{tr}}^{2}-\varepsilon_{\mathrm{tr} 0}^{2}}{\varepsilon_{\mathrm{tr} 0}^{2}}=\beta_{0}^{2} k^{2}\left(\frac{\sigma_{p}}{p_{0}}\right)^{2}, \\
\varepsilon_{\mathrm{ph}}^{2}-\varepsilon_{\mathrm{ph} 0}^{2}=0,
\end{gathered}
$$

where the subscript 0 corresponds now to the lens entrance and no subscript corresponds to the lens exit. Once more, independence between transverse and longitudinal distributions is assumed.

Equation (17) expresses the well-known chromaticity effect. Because of the energy spread, there is a jump of the trace emittance when crossing a focusing element. To limit this jump, $k_{0}^{2}$ should be the lowest (smoothest focusing) and $\beta_{0}^{2}$ (or the beam size) should be the smallest. Notice that the latter condition can be met by minimizing the Twiss parameter $\gamma$ in the drift preceding the lens. Taking into account the same result highlighted in the preceding paragraph, minimizing $\gamma$ at the plasma exit is the key point: It is doubly beneficial, for minimizing phase emittance growth when going through a free drift and also minimizing trace emittance growth when crossing a thin lens. 
Typically, it is necessary to focus the divergent beam $\left(\alpha_{0}<0\right)$ coming from the plasma to make at least a parallel beam $(\alpha=0)$. For that, using the definitions of the Twiss parameters in Eqs. (4)-(6), $\overline{x x^{\prime}}$ must be canceled, and Eq. (C2) shows that

$$
k_{0}=\frac{\alpha_{0}}{\beta_{0}} .
$$

Combining with Eq. (18) leads to

$$
\frac{\varepsilon_{\mathrm{tr}}^{2}-\varepsilon_{\mathrm{tr} 0}^{2}}{\varepsilon_{\mathrm{tr} 0}^{2}}=\alpha_{0}^{2}\left(\frac{\sigma_{p}}{p_{0}}\right)^{2},
$$

which means that the least jump in trace emittance can be obtained for a minimum $\alpha$ at the lens entrance, and, once more, this is met for a minimum $\gamma$ in the drift upstream from the lens.

Sections II A, II B, and II C analyzed the relations linking trace and phase emittances, and then their evolutions through a free drift and a thin lens, under the assumption of uncoupled transverse and longitudinal motion. Thanks to this simplified context, the main behaviors of the two emittances are pointed out with a single formalism, and all the parameters governing their evolutions are highlighted: the emittances at the plasma exit, the Twiss parameter $\gamma$ at the drift entrance, and the length and smoothness of the transfer line. As no other parameter than those ones is involved, we can thereafter focus on the mastering of these parameters, and only of them, to minimize emittance growth.

Before all nevertheless, it is necessary to check the validity and the accuracy of the above findings in a more general framework, with less restrictive assumptions. We propose, in a first step, to use analytical calculations on a general transfer line to confirm the emittance behaviors pointed above and, in a second step, to use numerical tracking through free drifts and finite-length quadrupoles to quantify the relevance of formulas (13), (14), (17), and (18). That will be presented, respectively, in Sec. IID and IIE in the following.

\section{Emittance evolution through a general transfer line}

If space charge effects can be neglected, the transfer line can be modeled by a first-order transfer matrix $R$ and higher-order tensors $T$ and $U$ :

$$
X=R \cdot X_{0}+T\left(X_{0}, X_{0}\right)+U\left(X_{0}, X_{0}, X_{0}\right)
$$

or with Einstein notation:

$$
X_{i}=R_{i j} X_{0, j}+T_{i j k} X_{0, j} X_{0, k}+U_{i j k l} X_{0, j} X_{0, k} X_{0, l},
$$

where $X=\left(x, x^{\prime}, y, y^{\prime}, l, d p / p_{0}\right)$ for the trace emittance or $X=\left(x, p x, y, p y, d E / p_{0} c\right)$ for the phase emittance. Note that the tensors $R, T$, and $U$ are different according to the trace or phase coordinates.

The emittance is linked to the determinant of the sigma matrix (or covariance matrix) which is defined as

$$
\Sigma=\overline{X \cdot X^{T}},
$$

of which the linear part, noted $\tilde{\Sigma}$, can be expressed in terms of $\Sigma_{0}$, the sigma matrix at entrance, as

$$
\tilde{\Sigma}=R \cdot \Sigma_{0} \cdot R^{T}
$$

The expansion of $\Sigma$, as defined in Eq. (23), gives, if we assume a symmetric distribution where first- and thirdorder moments are assumed to be zero,

$$
\begin{aligned}
\Sigma= & \tilde{\Sigma}+\left(T_{i k l} T_{j m n}+R_{i k} U_{j l m n}+R_{j k} U_{i l m n}\right) \\
& \times\left\langle X_{k} X_{l} X_{m} X_{n}\right\rangle_{0}+o\left(\Sigma_{0}^{3}\right) .
\end{aligned}
$$

In the case of a Gaussian distribution, we have

$$
\left\langle X_{k} X_{l} X_{m} X_{n}\right\rangle_{0}=\Sigma_{0, k l} \Sigma_{0, m n}+\Sigma_{0, k m} \Sigma_{0, l n}+\Sigma_{0, k n} \Sigma_{0, l m} .
$$

It is shown in Ref. [21] that Eq. (25) can be written

$$
\begin{aligned}
\Sigma_{i j}= & R_{i k} R_{j l} \Sigma_{0, k l}+\left[T_{i k l} T_{j m n}+2 T_{i k m} T_{j l n}\right. \\
& \left.+3\left(R_{i k} U_{j l m n}+R_{j k} U_{i l m n}\right)\right] \Sigma_{0, k l} \Sigma_{0, m n}+o\left(\Sigma_{0}^{3}\right) .
\end{aligned}
$$

In the more general case of a non-Gaussian distribution, but with a zero final dispersion and assuming that the transverse and longitudinal distribution are uncorrelated, when considering only the chromatic terms in $T$ and $U$, we get

$$
\begin{aligned}
\Sigma_{i j} \approx & \tilde{\Sigma}_{i j}+\left(T_{i k l} T_{j 66}+T_{i 66} T_{j k l}+4 T_{i k 6} T_{j l 6}\right. \\
& \left.+3 R_{i k} U_{j l 66}+3 R_{j k} U_{i l 66}\right) \Sigma_{0, k l} \Sigma_{0,66}+o\left(\Sigma_{0}^{3}\right) .
\end{aligned}
$$

The calculation of the determinant of the $\Sigma$ matrix gives then for the horizontal plane (the vertical plane is deduced by replacing indexes 1 and 2 by, respectively, 3 and 4)

$$
\begin{aligned}
\frac{\varepsilon^{2}-\varepsilon_{0}^{2}}{\varepsilon_{0}^{2}} \approx & {\left[\gamma\left(2 T_{1 k l} T_{166}+4 T_{1 k 6} T_{116}+6 R_{1 k} U_{1 l 66}\right)+\beta\left(2 T_{2 k l} T_{266}+4 T_{2 k 6} T_{2 l 6}+6 R_{2 k} U_{2 l 66}\right)\right.} \\
& \left.+2 \alpha\left(T_{1 k l} T_{266}+T_{2 k l} T_{166}+4 T_{1 k 6} T_{2 l 6}+3 R_{1 k} U_{2 l 66}+3 R_{2 k} U_{1 l 66}\right)\right] \frac{\Sigma_{0, k l}}{\varepsilon_{0}} \Sigma_{0,66}+o\left(\Sigma_{0,66}\right) .
\end{aligned}
$$


From this general formula, in the case of a drift of length $l$, where $T_{126}=T_{346}=l / 2 \beta_{0}$ and $U_{1266}=U_{3466}=\frac{3-\beta_{0}^{2}}{6 \beta_{0}^{2}} l$, Eq. (15) can be deduced when using the canonical coordinates: $\frac{\varepsilon_{\mathrm{ph}}^{2}-\varepsilon_{\mathrm{ph} 0}^{2}}{\varepsilon_{\mathrm{ph} 0}^{2}} \approx \gamma_{0}^{2} l^{2} \frac{\sigma_{p}^{2}}{p_{z}^{2}}$.

In the case of a thin lens of integrated gradient $k$ where $T_{216}=T_{436}=k / 2$ and $U_{2166}=U_{4366}=-k / 3$, Eq. (17) can be deduced when using the coordinates of the trace emittance: $\frac{\varepsilon_{\mathrm{tr}}^{2}-\varepsilon_{\mathrm{tr} 0}^{2}}{\varepsilon_{\mathrm{tr} 0}^{2}} \approx \beta_{0}^{2} k^{2}\left(\frac{\sigma_{p}}{p_{0}}\right)^{2}$.

If now the coordinates for the phase emittance are used, then $T_{126}=T_{346}=0$, implying that the emittance is not changed by the thin lens as given in Eq. (18).

This section shows that it is possible to estimate analytically the emittance growth via transfer matrix terms of whatever a transfer line. This global approach allows us to confirm the evolutions of the two emittances pointed out precedently in restrictive conditions. Even more importantly, Eq. (29) is used thereafter in Sec. IV in the optimization of the transfer line aiming at minimizing emittance growth. Such an analytical expression allows one to significantly speed up the time-consuming numerical procedure.

\section{E. Particle tracking}

Simulations with the particle tracking code TraceWin [22] are undertaken so as to check the validity of the formulas established above. For that, a simple structure including a $0.1 \mathrm{~m}$ drift followed by a $0.01 \mathrm{~m}$ quadrupole and then a second drift is considered. The electron beam at the start has the following features: $10^{4}$ macroparticles in a Gaussian distribution cut at 6 sigmas (thus, transverse and longitudinal coordinates are not strictly independent, but the dependence is weak). The Twiss parameters are $\alpha_{x_{0}}=-40, \beta_{x_{0}}=1 \mathrm{~m}$, and $\varepsilon_{\text {trx } x_{0, n}}=10^{-6} \mathrm{mrad}$ in transverse and $\alpha_{z_{0}}=0, \quad \beta_{z_{0}}=1 \mathrm{~m}$, and $\varepsilon_{\text {tr }_{0, n}}=$ $0.01 \times 10^{-6} \mathrm{mrad}$. This corresponds to the rms sizes $\sigma_{x_{0}}=10^{-5} \mathrm{~m}, \sigma_{x 0}^{\prime}=4 \times 10^{-4} \mathrm{rad}$, and $\frac{\sigma_{p}}{p_{0}}=1 \%$.

The resulting variations of the beam size, the trace, and the phase emittances are shown along the structure in Fig. 1 for three different quadrupole integrated gradients $G l=330,130$, and $100 \mathrm{~T}$. The second gradient is chosen so as to have a parallel beam ( $\alpha=0)$ after focusing. We can observe the following. (i) The trace emittance is constant in the drifts and experiences an abrupt jump in the quadrupoles, a jump that is smaller for lower Gl. (ii) The phase emittance increases in the drifts but only when the beam size is varying, whether it is convergent or divergent, and varies very little in the quadrupole. (iii) The normalized trace emittance and phase emittance are equal only and everywhere $\alpha=0$. This occurs at two precise locations for $G l=330 \mathrm{~T}$ or on a long distance after the quadrupole for $G l=130 \mathrm{~T}$, whereas for smaller focusing strengths the two emittance curves never cross as the beam has no waist.
Those behaviors are totally in agreement with the formulas given previously. Quantitatively, the discrepancies between these formulas and numerical tracking are less than $15 \%$ (see Table I), although there is a weak dependence between transverse and longitudinal coordinates, and the quadrupole has a finite length.

\section{F. The three key roles}

The above studies show precisely the relation between the two emittances, which emittance grows in which context, and the exact parameters governing these growths. By means of a unique and consistent formalism, the four equations (13), (14), (17), and (18) show the following. (i) Through a free drift, the trace emittance is unchanged, while the phase emittance regularly grows along the drift, all the more so for bigger Twiss parameter $\gamma_{0}$ at the drift entrance $\left(\gamma_{0}\right.$ remaining anyway constant in the drift). (ii) Through a thin lens, the phase emittance is unchanged, while the trace emittance is subjected to a kick, and that more considerably when the focusing strength is bigger.

Those behaviors have been above supported by more general analytical considerations and quantitatively checked with multiparticle tracking.

Even more importantly, the above studies highlight exhaustively the primary parameters governing emittance growth when transferring the accelerated beam from a plasma stage to another plasma stage or toward the users. Therefore, we can focus exclusively on them for mitigating the emittance growth. Furthermore, this analysis points out the only location where it is possible to act on those parameters. All that allows us to announce the three tasks necessary for mitigating emittance growth, associated to the three key roles: (1) minimizing emittance and energy spread during acceleration-therefore, this should be the exclusive role of the acceleration plasma; (2) minimizing $\gamma_{0}$ (Twiss parameter) at the transfer line entrance-therefore, this should be the exclusive role of the plasma down ramp, with the reservation that the latter would not itself induce significant emittance growth; (3) minimizing the total length and the integrated focusing strength in the transfer line-therefore, this should be the exclusive role of the focusing elements in the transfer line.

Once the best is achieved at each of those three components as recommended, the emittance growth is ensured to be the minimum. The advantage is that the optimization can be done separately at each stage without minding about the nature or the capacity of the next stage. On the contrary, if it is not done correctly at a given stage, it cannot be compensated elsewhere. That is why it is important to insist on the exclusive role of each stage.

In the following, we examine a given case of laserplasma acceleration where the emittance and energy spread are already minimized along the acceleration process and only the two last tasks listed above will be then successively explored. 
(a)

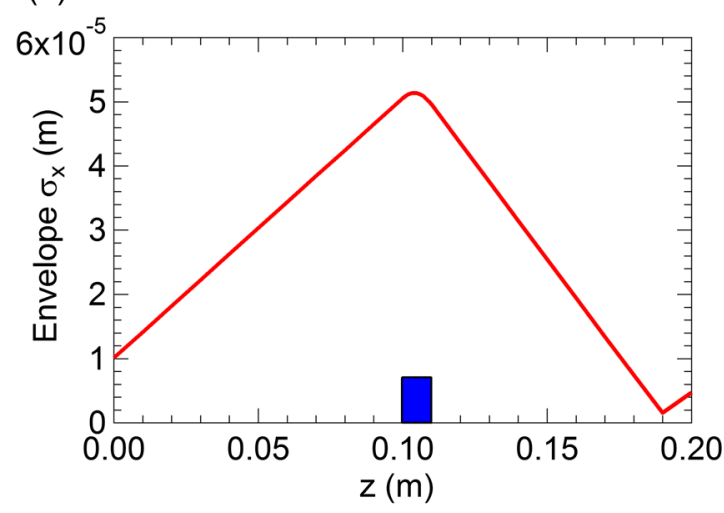

(b)

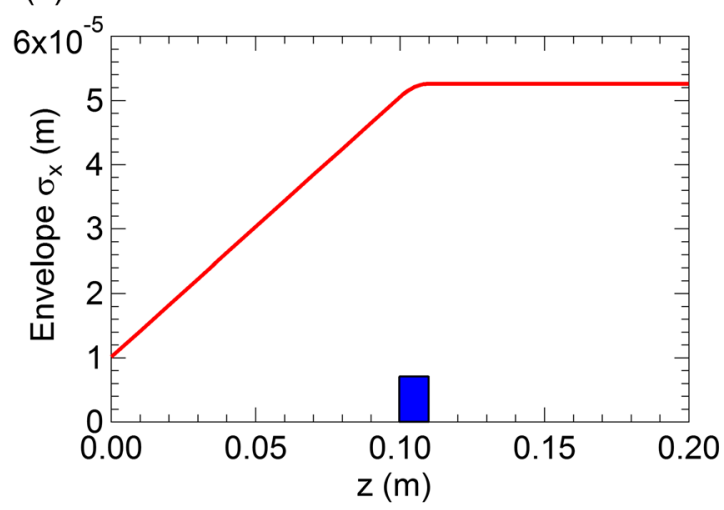

(c)

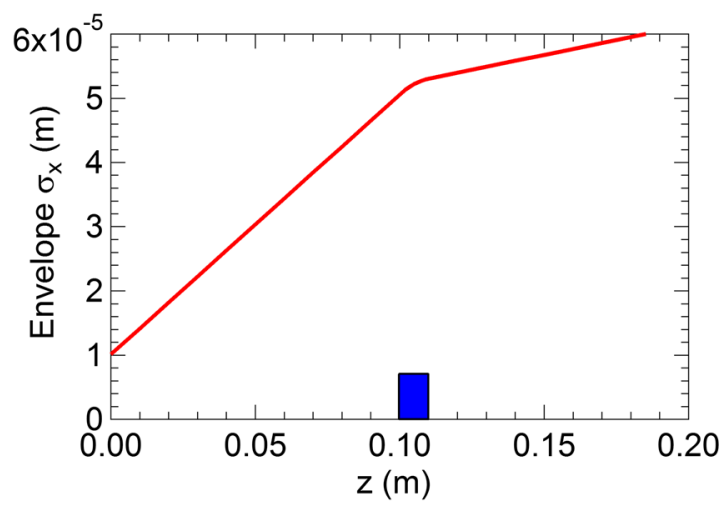

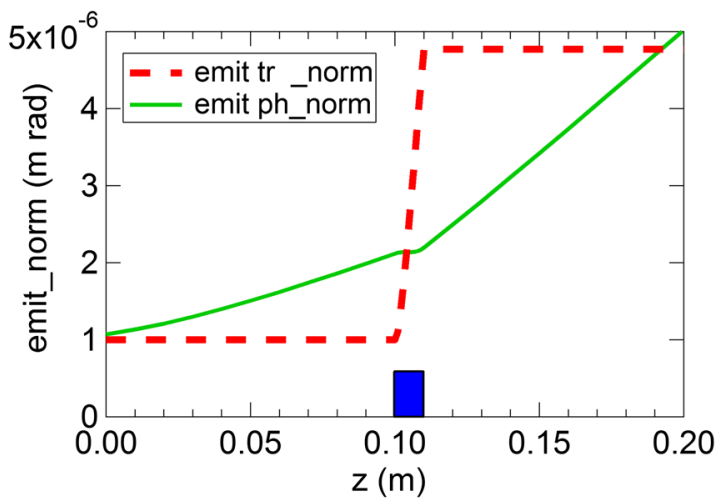
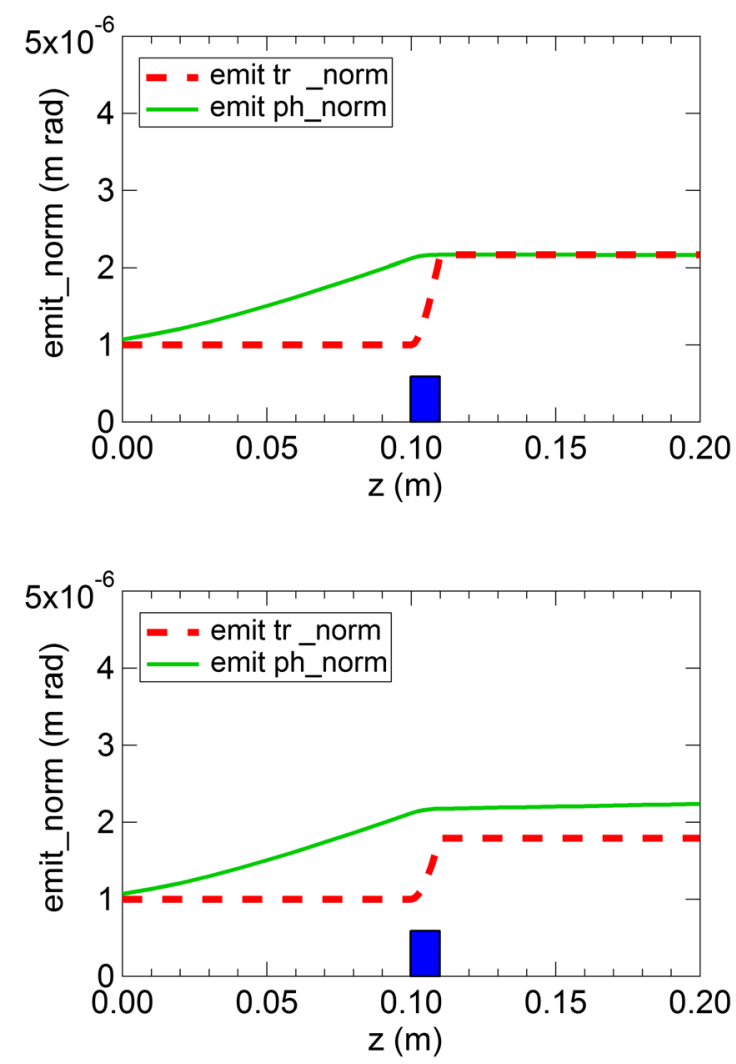

FIG. 1. Variation of beam size (left), normalized phase and trace emittance (right) along a structure including a $0.1 \mathrm{~m}$ free drift, followed by a $0.01 \mathrm{~m}$ quadrupole (blue rectangle in the graphs) and a second drift. The cases of three different quadrupole strengths are shown from top to bottom: 330 (a), 130 (b), and $100 \mathrm{~T}$ (c), the second one being chosen for obtaining a parallel beam after focusing.

TABLE I. Emittance variation through the first drift or through the quadrupole, given by the formulas or the particle tracking.

\begin{tabular}{lcc}
\hline \hline Emittance variation $\left(10^{-12} \mathrm{~m}^{2} \mathrm{rad}^{2}\right)$ & $\begin{array}{c}\text { Eq. (14) } \\
\text { or (17) }\end{array}$ & Tracking \\
\hline$\varepsilon_{\mathrm{ph}}^{2}-\varepsilon_{\text {ph0 }}^{2}$ when crossing the first drift & 3.76 & 3.34 \\
$\varepsilon_{\text {tr }}^{2}-\varepsilon_{\text {tr0 }}^{2}$ when crossing quadrupole $330 \mathrm{~T}$ & 5.50 & 5.72 \\
$\varepsilon_{\text {tr }}^{2}-\varepsilon_{\text {tr0 }}^{2}$ when crossing quadrupole $130 \mathrm{~T}$ & 3.72 & 3.68 \\
$\varepsilon_{\text {tr }}^{2}-\varepsilon_{\text {tr0 }}^{2}$ when crossing quadrupole $100 \mathrm{~T}$ & 2.18 & 2.40 \\
\hline \hline
\end{tabular}

\section{LIMITING EMITTANCE GROWTH WITH PLASMA RAMPS}

\section{A. Laser-plasma configuration}

We consider here the laser-plasma acceleration stage of the EuPRAXIA project [10]. The plasma, at the nominal density $n_{p 0}$, consists of three sections: (i) a matching-in or up ramp section to match the coming electron beam into the plasma channel, with a density increasing from zero to $n_{p 0}$, (ii) an acceleration section to boost the beam energy from $150 \mathrm{MeV}$ to $5 \mathrm{GeV}$, with a longitudinally uniform density 
TABLE II. Main parameters of the plasma acceleration section.

\begin{tabular}{|c|c|c|}
\hline \multicolumn{3}{|l|}{ Laser } \\
\hline Strength $a_{0}$ & \multicolumn{2}{|c|}{2.0} \\
\hline Spot size $k_{p} w_{0}$ & \multicolumn{2}{|c|}{3} \\
\hline Pulse length $k_{p} \sigma_{z}$ & \multicolumn{2}{|c|}{1} \\
\hline $\begin{array}{l}\text { Plasma } \\
\text { Density } n_{p 0} \\
\text { Channel depth } \Delta n / \Delta n_{c} \\
\text { Plateau length }\end{array}$ & \multicolumn{2}{|c|}{$\begin{array}{c}1 \times 10^{17} \mathrm{~cm}^{-3} \\
0.35 \\
260 \mathrm{~mm}\end{array}$} \\
\hline Electron beam & $\begin{array}{c}\text { Before } \\
\text { acceleration }\end{array}$ & $\begin{array}{c}\text { After } \\
\text { acceleration }\end{array}$ \\
\hline Charge $Q_{b}$ & $30 \mathrm{pC}$ & $30 \mathrm{pC}$ \\
\hline Energy $E_{k}$ & $150 \mathrm{MeV}$ & $5 \mathrm{GeV}$ \\
\hline Energy spread $\sigma_{E} / E$ & $0.5 \%$ & $1.1 \%$ \\
\hline Slice energy spread $\sigma_{E_{s}} / E$ & $0 \%$ & $0.09 \%$ \\
\hline $\begin{array}{l}\text { rms emittance } \varepsilon_{\mathrm{ph}, n} \\
\operatorname{rms} \text { size } \sigma\end{array}$ & $\begin{array}{c}1.0 \mathrm{~mm} \mathrm{mrad} \\
1.3 \mu \mathrm{m}\end{array}$ & $\begin{array}{c}1.03 \mathrm{~mm} \mathrm{mrad} \\
0.5 \mu \mathrm{m}\end{array}$ \\
\hline Twiss parameter $\gamma$ & $2003 \mathrm{~m}^{-1}$ & $411 \mathrm{~m}^{-1}$ \\
\hline rms length $\sigma_{z}$ & $2.1 \mu \mathrm{m}$ & $2.1 \mu \mathrm{m}$ \\
\hline
\end{tabular}

$n_{p 0}$, and (iii) a matching-out or down ramp section to match the ultrasmall beam to the following transport beam line, with a density decreasing from $n_{p 0}$ to zero. Throughout these sections, the beam quality needs to be preserved as much as possible, so that it can be used to drive an x-ray free-electron laser. The acceleration section has been previously studied, showing that the beam emittance could be maintained during the acceleration by matching the beam size to the wakefield focusing gradient [23], and the beam energy spread along with the slice energy spread can be controlled by manipulating the beam loading effect adequately [24]. The resulting optimized parameters of the laser, the plasma, and the beam before and after acceleration are summarized in Table II, where $k_{p}$ is the plasma wave number and $n_{c}$ is the critical channel depth [23].

At the entrance of the acceleration section, the beam must present at a waist with a very large Twiss parameter $\gamma$, corresponding to a tiny transverse size. This will need a very strong focusing in the upstream transfer line, leading to a significant emittance growth according to Eq. (17). Adding a plasma up ramp is mandatory to ease these requirements at injection.

At the end of the acceleration section, due to the very strong focusing provided by the wakefield, the beam size is very small, associated to a very big divergence, characterized by the large Twiss parameter $\gamma$. If the plasma ended sharply at this position, the beam emittance will strongly grow in the next transfer line, according to Eqs. (14) and (17). Adding a plasma down ramp will help to efficiently mitigate this issue.

\section{B Matching in with plasma density up ramp}

In order to mitigate the emittance growth by the betatron decoherence mechanism [13], the incoming electron beam must be properly matched to the focusing structure of the plasma acceleration stage where the plasma density is constant (longitudinally). This means its Twiss parameters must verify

$$
\alpha=0 \quad \text { and } \quad \beta=\frac{1}{\sqrt{K}},
$$

where $K$ is the focusing gradient at the plateau entrance. As the latter is very large, the beam transverse size is tiny, of the order of $1 \mu \mathrm{m}$. This requires very strong focusing strengths in the upstream transfer line, inducing significant emittance growth according to Eq. (17). It is thus mandatory to implement a density up ramp to smoothly increase the plasma density from zero to its value in the plateau in order to relax the two constraints above.

We set ourselves the objective to relax the required beam size to $10 \mu \mathrm{m}$ and propose to explore the Twiss parameter $\alpha_{0}$ at the ramp entrance and the ramp length $L$ so that there is a minimum emittance growth, for exponential up ramps with density defined as

$$
n_{p}(z)=n_{p 0} \exp (z / L)
$$

over $-5 L<z<0$.

Instead of a fully 3D particle-in-cell (PIC) simulation, we first use the electromagnetic fields calculated from linear theories to replace the laser wakefield and the beam loading effect for the tracking of the electron beam, as done in the Astra code [25], so that a fast parameter scan is possible. The results are shown in Fig. 2. It is found that, for each up ramp length, there exists an optimal $\alpha_{0}$ that results in a minimum emittance growth. The longer the ramp is, the less the

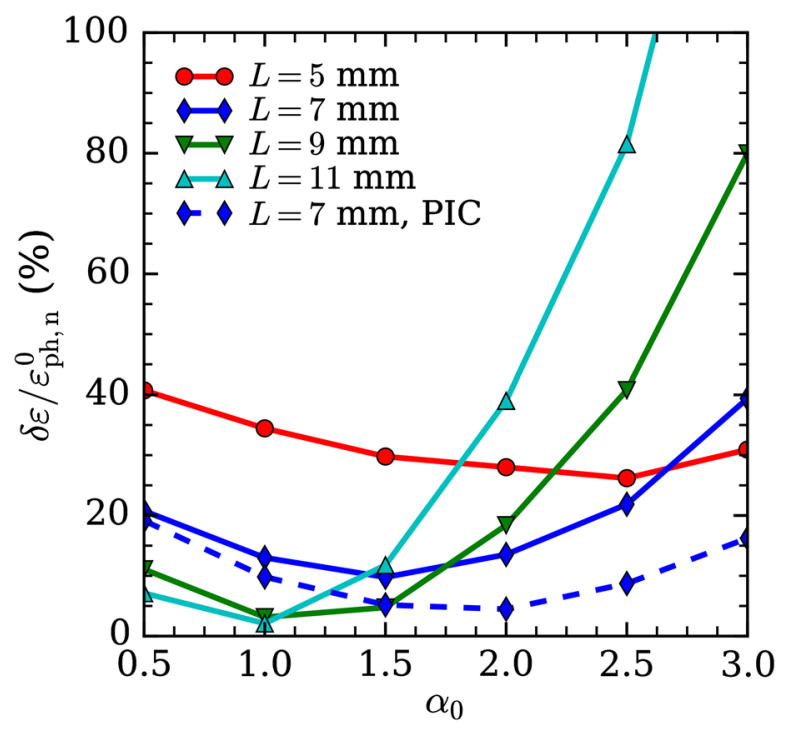

FIG. 2. Emittance growth as a function of $\alpha_{0}$ for various ramp lengths, calculated with the Astra code (continuous lines) and one case calculated with the 3D PIC code Warp (dashed line). 
emittance grows, but the minimum is more peaked, meaning that it should be more precisely achieved. In the cases studied here, the minimum emittance growth is found to be around $2 \%$, with a ramp length of $L=11 \mathrm{~mm}$ and $\alpha_{0}=1.0$.

Then 3D PIC simulations were conducted with the Warp code in the boosted frame [26], with the boosted factor $\gamma_{\text {boost }}=16$. Since here the laser pulse is not focused by any external means, its spot size evolves following

$$
w(z)=w_{0} \sqrt{1+z^{2} / z_{R}^{2}},
$$

where $z_{R}=\pi w_{0}^{2} / \lambda_{0}$ is the Rayleigh length and $\lambda_{0}$ the laser wavelength. As a result, the transverse size of the moving window in the simulation is much larger than that used in the acceleration stage where the laser pulse is focused by the parabolic plasma channel. The dimension of the moving window is typically $404 \times 404 \times 150 \mu \mathrm{m}$ with $466 \times$ $466 \times 5524$ cells and only one particle per cell for the plasma and about 200 particles per cell for the electron beam. In order to speed up simulations, the plasma particle per cell here is set to only 1 . Nevertheless, 3D simulations has been compared to 2D simulations in cylindrical coordinates with many more plasma particles per cell, and a good agreement has been found in terms of the laser pulse evolution and the longitudinal wakefield distribution.

In order to save computation time, a not too long ramp, $L=7 \mathrm{~mm}$, is studied. It is found that (see Fig. 2) results are even better than those found with the Astra code for the same ramp length: For $\alpha_{0}=2.0$, emittance growth is only $4 \%$. The evolutions along the up ramp of beam emittance and beam size are also shown in Fig. 3. It is interesting to note that, no matter how well the beam is matched, the beam size always converges to its matched value, due to the presence of the very strong transverse focusing gradient. But the stronger the beam size oscillates before it reaches its equilibrium value, the more the emittance increases.

\section{Matching out with plasma density down ramp}

A plasma density decreasing smoothly to zero is highly desirable instead of a sharp decrease. We will consider in the following different types of density profile, exponential, linear, and adiabatic, and for each of them will adjust their length so as to minimize the parameter $\gamma$ at the ramp exit as recommended in Sec. II.

3D PIC simulations have been carried out with the Warp code in the boosted frame [26], under the similar setup of the simulation area and grid size as in Sec. III B. Figure 4 shows the evolution of $\gamma$ and emittance till far in the free drift downstream from the plasma, for the exponential profile defined as

$$
n_{p}(z)=n_{p 0} \exp (-z / L)
$$

over $0<z<5 L$, for $L=3,5,7 \mathrm{~mm}$. The longer the ramp, the smaller is $\gamma$, and consequently the phase emittance varies less after the ramp as predicted by Eq. (14), but then emittance growth inside the ramp itself becomes more significant.

Notice that the emittance growth occurs only near the ramp exit. This is due to the presence of the beam loading effect, i.e., due to nonlinear focusing fields excited by the beam itself. As pointed out in Ref. [23], when the wakefield is strong enough, the beam loading effect could be negligible. In the down ramp, however, the wakefield decreases due to both the reduced (or increased) laser strength (or spot size) and the reduced plasma density, while the beam loading effect decreases mainly due to the

(b)

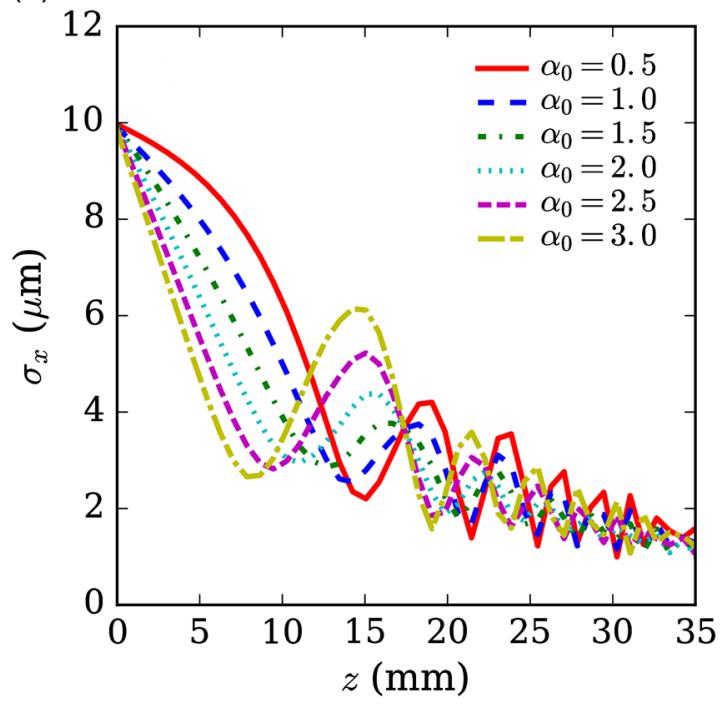

FIG. 3. Beam emittance (a) and size (b) evolutions in the up ramps. 
(a)

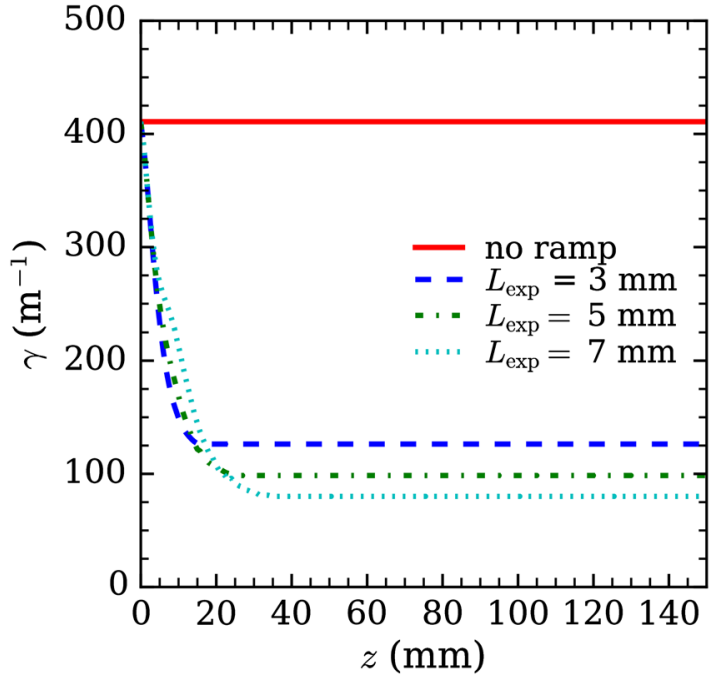

(b)

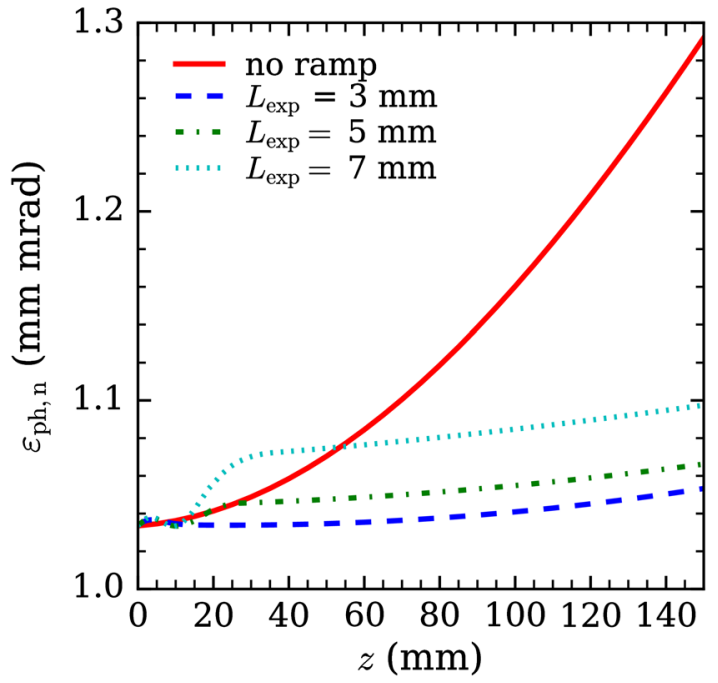

FIG. 4. Evolution of Twiss parameter $\gamma$ (a) and normalized phase emittance $\varepsilon_{\mathrm{ph}, n}$ (b) along the down ramp and then the downstream drift, for the exponential density profiles with $L=3,5$, and $7 \mathrm{~mm}$, compared to the case without a ramp. $z=0 \mathrm{~mm}$ is the down ramp entrance.

reduced plasma density. The wakefield is further decreased in the down ramp, because it is no longer in the resonant conditions set in the plasma plateau [23]. It means that, at some point, the beam loading effect may prevail over the wakefield, and as a result the nonlinear focusing field becomes dominant, degrading quickly the beam quality. In our simulations, the plasma density was reduced to zero too fast for the beam loading effect to influence the beam quality when the down ramp is short (e.g., $3 \mathrm{~mm}$ ). But for a longer down ramp (e.g., 5-7 mm), the beam loading effect started to deteriorate the beam quality from the middle of the ramp, leading to a valuable emittance growth. Therefore, the down ramp length should be chosen in such a way that the emittance is not affected too much by the beam loading effect, while $\gamma$ is small enough not to affect the emittance in the following transfer line.

Linear density profiles have also been tried (not shown here), and it is found that, for a length around $3 L$, equivalent results are obtained while being slightly less good when compared to exponential profiles.

All those results show that a smooth down ramp with the appropriate length, which must not be at a very precise value, thus easily achieved, is enough to strongly mitigate emittance growth in the downstream transfer line, compared to the case without a down ramp. From the practical point of view, such plasma density ramps can also be implemented with a good flexibility, for example, by changing the plasma cell radius at appropriate positions at the cell entrance and exit $[16,27]$.

We have also wondered whether an adiabatic down ramp as recommended by Ref. [14] could help avoid emittance growth in the down ramp itself. That could be achieved with a focusing strength evolving as

$$
K(z)=\frac{K_{0}}{(1+g z)^{4}}
$$

where $K_{0}$ is the focusing strength at the ramp entrance and $g$ is a parameter to adjust the ramp length. The generation of such a profile has been proposed by tapering the laser spot size in a constant density plasma [15], resulting in a preserved emittance being observed for a very low bunch charge. In this article, we propose to study a varying plasma density in the presence of a laser spot size without any specific focusing technique in order to produce such an adiabatic profile. After some first tries, $g=100 \mathrm{~m}^{-1}$ seems to be the most appropriate value. Figure 5 gives the adiabatic profile $K(z)$ and the corresponding density profile, compared to an exponential profile with $L=7 \mathrm{~mm}$. The adiabatic density profile drops very quickly and then increases gradually to around $10 \%$ of the initial density. That is because the laser spot size (strength) has become so large (small) that the plasma density has to be increased to maintain the gradient profile of Eq. (34). As a result, the adiabatic focusing decreases faster at the beginning than at the end, compared to that of the exponential profile.

The Twiss parameter $\gamma$ and the phase emittance growth in the presence of the adiabatic down ramp are given in Fig. 6. Compared to the case of an exponential down ramp, $\gamma$ is here even smaller, but the emittance growth is much more significant. As discussed above, this emittance growth comes from the nonlinear fields generated by the beam loading effect that become significant in the down ramp as the plasma density decreases. A way to avoid it is to cut the adiabatic density profile earlier, letting it going down quickly to zero after $z=10 \mathrm{~mm}$, for example. The beam would then be extracted out of the ramp before its emittance gets worse, but the Twiss parameter $\gamma$ would be still large. 

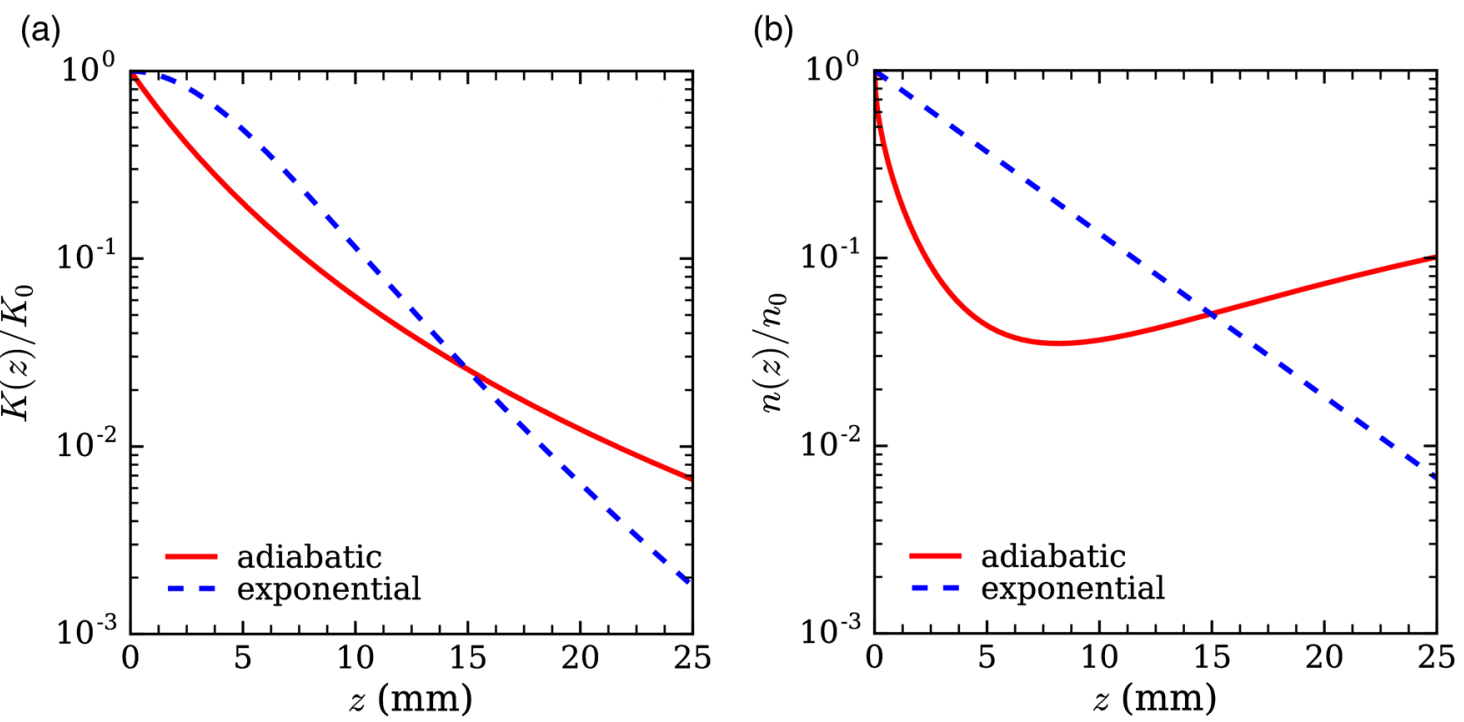

FIG. 5. Focusing gradient (a) and corresponding plasma density profiles (b) for adiabatic $\left(g=100 \mathrm{~m}^{-1}\right)$ and exponential $\left(L_{\exp }=7 \mathrm{~mm}\right)$ down ramps.

Only in the case of a very small bunch charge, $0.3 \mathrm{pC}$ as shown in Fig. 6 and in Ref. [15], is the beam loading effect negligible and can the emittance be kept unchanged. In any case, the adiabatic density profile as studied here is not very practical to achieve. Linear or exponential down ramps would be preferable, regarding the ease of its implementation and the efficiency of its emittance growth mitigation.

\section{Discussions}

Plasma down ramps are very efficient for decreasing the Twiss parameter $\gamma_{0}$, as recommended in Sec. II for minimizing emittance growth in the transfer line next to the plasma acceleration stage. Furthermore, no specific density profile is really needed, as long as the ramp length is correctly chosen, and this can be done without the need of great precision. The longer the down ramp, the smaller $\gamma_{0}$ is, but then emittance growth in the ramp itself caused by the nonlinear effect progressively counterbalances the benefit of smaller $\gamma_{0}$. As the latter mechanism is rather progressive, the optimum zone is smooth, meaning an easy achievement of the down ramp.

Up ramp plasmas are mandatory for properly matching the incoming beam into the plasma acceleration stage. Here also, no specific shape and no great precision are needed, as long as the ramp length and the beam divergence are correctly tuned. (a)

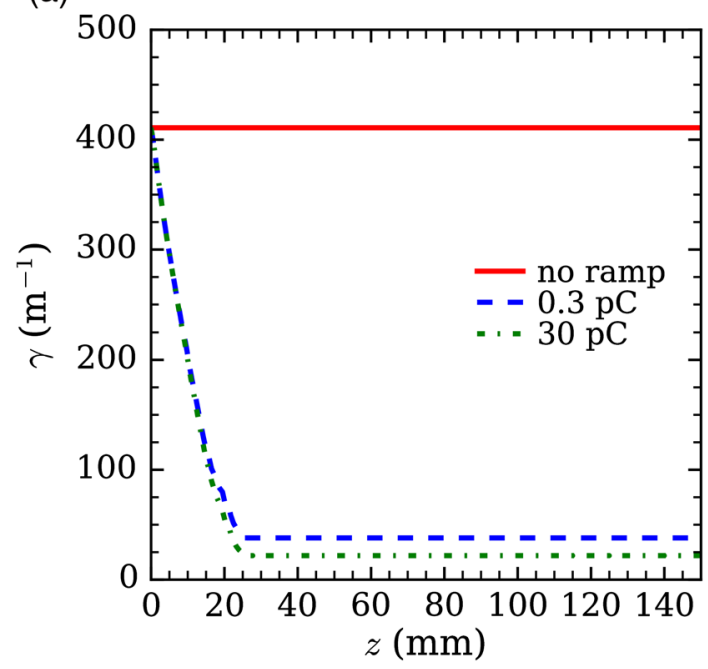

(b)

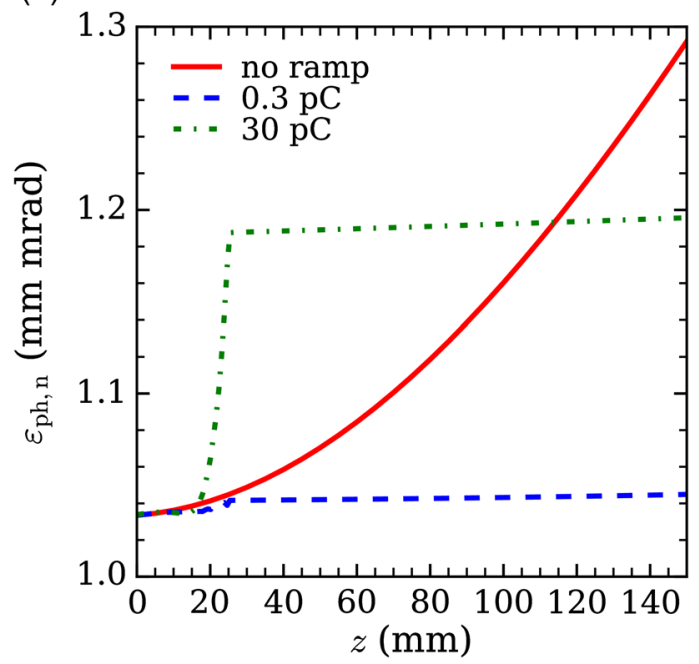

FIG. 6. Evolution of Twiss parameter $\gamma$ (a) and normalized phase emittance $\varepsilon_{\mathrm{ph}, n}$ (b) along the down ramp and then the downstream drift, for the adiabatic density profiles $\left(g=100 \mathrm{~m}^{-1}\right)$, compared to the case without a ramp. The case of a very low particle charge $(0.3 \mathrm{pC})$ is also shown. $z=0 \mathrm{~mm}$ is the down ramp entrance. 
TABLE III. Characteristics of the electron beam obtained at the down ramp exit and as required at the FEL entrance.

\begin{tabular}{lcc}
\hline \hline Electron beam & Down ramp exit & FEL entrance \\
\hline rms emittance $\varepsilon_{\mathrm{ph}, n}$ & $1.07 \mathrm{~mm} \mathrm{mrad}$ & $\sim 1 \mathrm{~mm} \mathrm{mrad}$ \\
rms size $\sigma$ & $2.8 \mu \mathrm{m}$ & $23 / 15 \mu \mathrm{m}$ \\
Twiss param. $\gamma$ & $80.1 \mathrm{~m}^{-1}$ & $0.63 / 0.67 \mathrm{~m}^{-1}$ \\
\hline \hline
\end{tabular}

\section{OPTIMIZING BEAM TRANSFER TO FEL ENTRANCE}

\section{A. Objective and setup}

The objective here is to drive the beam from the down ramp exit obtained in Sec. III B, with the beam parameters as given in Table III, toward the FEL entrance with precise required parameters as given in the same table. It can be seen that the emittance growth should be maintained as low as possible.

As three parameters should be achieved in the two transverse directions, the transfer line should be designed with six quadrupoles. They can be divided into two groups: The first group can be used for capturing the beam coming from the plasma which often presents a big divergence. The quadrupoles should thus be the shortest and the closest to the plasma exit. Permanent magnets are the best suitable for that. The second group can be used for tuning the beam size and divergence at FEL entrance. Four electromagnets are enough for that.

Therefore, with two permanent magnet quadrupoles and four electromagnet quadrupoles for achieving the six constraints in Table III, the solution found for the quadrupole gradients should be unique once their lengths, positions, and polarities are fixed and when considering only their linear effects as here. However, in order to ensure that there are no better configurations with other quadrupole lengths and positions, or even with more permanent

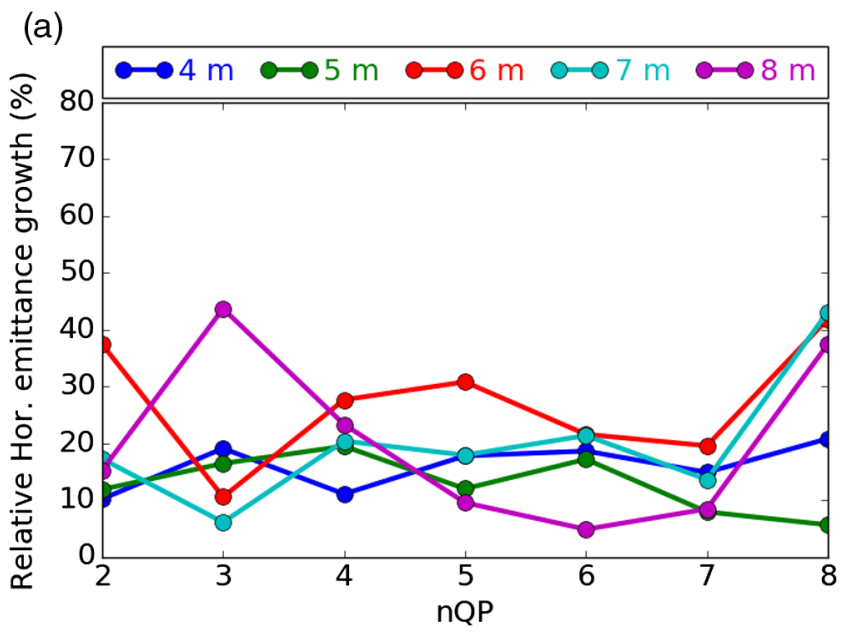

magnets as done in Ref. [20], we will also explore all those possibilities.

\section{B. Optimization method and results}

When the number of free parameters (unknowns) is larger than the number of constraints (equations), there are an infinity of solutions. For example, two close quadrupoles with whatever strengths produce the same effect as long as the difference of these strengths is the same. As the aim here is to search for the minimum of a function, that is of a surface in a multidimensional space, it is essential to find out the lowest minimum while avoiding the local minima. The particle swarm optimization (PSO) algorithm [28] is well suited for that, because it employs an "army" of "swarms" to explore the topology of the studied space before converging toward the lowest minimum.

A code based on the PSO algorithm has been written in Python to optimize the transfer line. When the program is near converging, a gradient conjugate method was used to speed up the convergence. Although requiring massive simulations, this optimization code is not very time consuming, because it relies uniquely on analytical transfer matrices like those used in Eq. (29) so that no particle tracking has to be performed. Only once the solution is found is the latter checked with the tracking codes TraceWin [22] and Astra [26], where space charge effects are taken into account.

The function to be minimized is the quadratic sum of the relative difference to the targeted Twiss parameters (as specified in Table III) and the relative emittance growth as specified in Eq. (29), which by its analytical nature allows one to significantly speed up numerical procedures. The unknown parameters to be determined are the position and the gradient of the quadrupoles. The fixed input data are the number of electromagnet quadrupoles (4), their length $(400 \mathrm{~mm})$, and their maximum gradient $(100 \mathrm{~T} / \mathrm{m})$; the

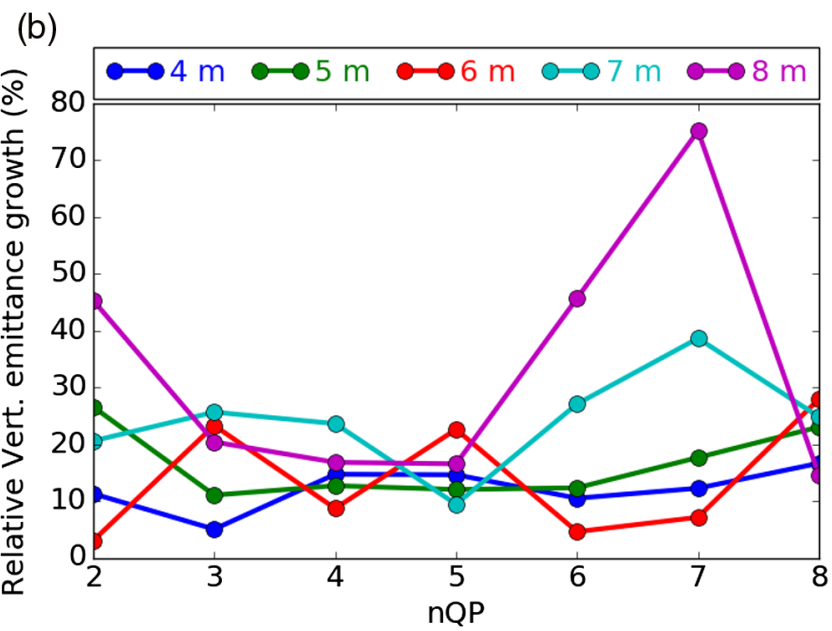

FIG. 7. Relative emittance growth [(a) horizontal and (b) vertical] as a function of the number of permanent quadrupoles and of the transfer line total length. 


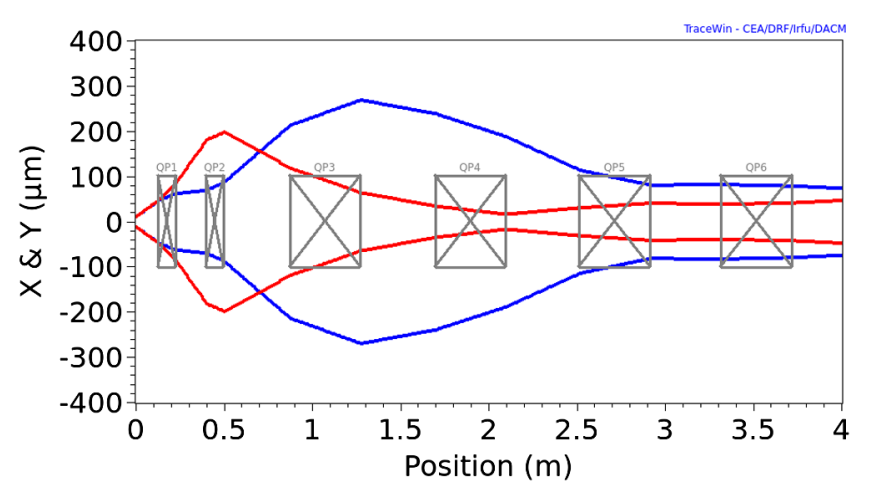

FIG. 8. 3-rms envelopes of the $5 \mathrm{GeV}$ beam in the transfer line from the plasma exit to the undulator entrance (blue, horizontal; red, vertical).

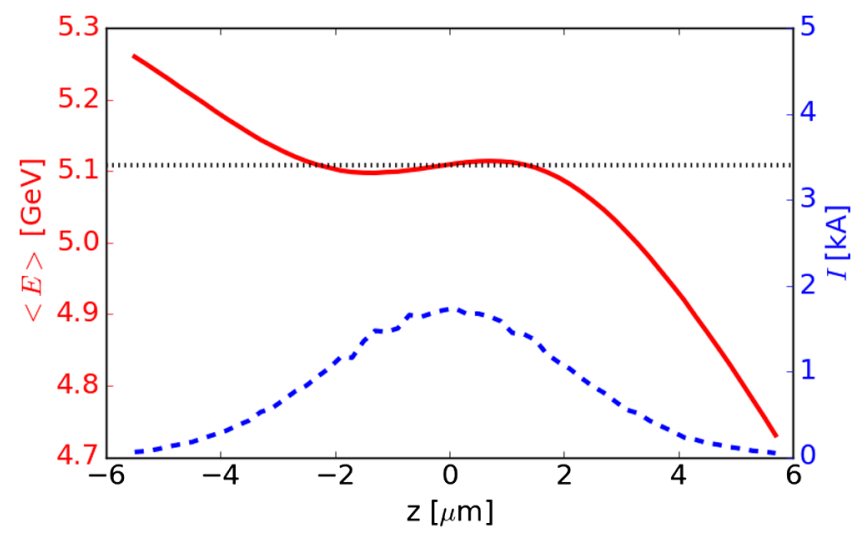

FIG. 9. Final beam current (blue dashed line) and average beam energy (red solid line) in each $0.2 \mu \mathrm{m}$ long slice. The reference energy is given by the black dotted line.

permanent magnet quadrupole length $(100 \mathrm{~mm})$ and maximum strength $(700 \mathrm{~T} / \mathrm{m})$; the distance between the first magnet and the plasma end $(50 \mathrm{~mm})$; and the minimum distance between the magnets $(30 \mathrm{~mm})$. The variable input data are the number of permanent magnets (from 2 to 8 ) and the total length of the transfer line (from 4 to $8 \mathrm{~m}$ ).

Results on phase emittance variations are shown in Fig. 7 as a function of the two last variable parameters. We can see that the results are roughly the same, proving that the PSO algorithm is capable of finding the lowest minimum, that is, the best solution, which is necessarily the unique solution when there are only two permanent magnets.

This study confirms that, as sensed at the end of Sec. IVA, because six parameters must be mastered, the solution with six quadrupoles is the best one. Once such a line is implemented, i.e., when the quadrupoles with given polarities and lengths are positioned, the solution is unique, so that classical algorithms searching by the largest slope method can be used for quickly finding out the minimum. By then changing either the quadrupole polarities, lengths, or positions, further satisfying solutions can be found.

Indeed, when more than six quadrupoles are used, i.e., more than two magnets are used in Fig. 7, often the maximum strength is higher and sometimes much higher, because, as said above, close magnets can contradict between them, whereas all other results remain the same. Besides, there is no significant difference between lengths of 4 and $5 \mathrm{~m}$, but, for longer lengths, the emittance growth becomes higher as predicted by Eq. (14) for a free drift. This growth looks, however, not so significant in Fig. 7, because the effect of the first drift is the most important, whereas all the cases studied here have the same first drift; for the following drifts, as asking for minimizing emittance growth is equivalent to asking for the smoothest focusing, it results in a bigger beam envelope in average and, thus, a smaller $\gamma$ in the drifts, reducing the effect of their lengths on emittance growth, always according to Eq. (14).

As a consequence, the shortest solution is adopted, with two permanent magnet quadrupoles and a total transfer length of $4 \mathrm{~m}$. The resulting beam envelope variation is shown in Fig. 8. The maximum gradient for the permanent magnet is $700 \mathrm{~T} / \mathrm{m}$ and for the electromagnet is $85 \mathrm{~T} / \mathrm{m}$. (a)

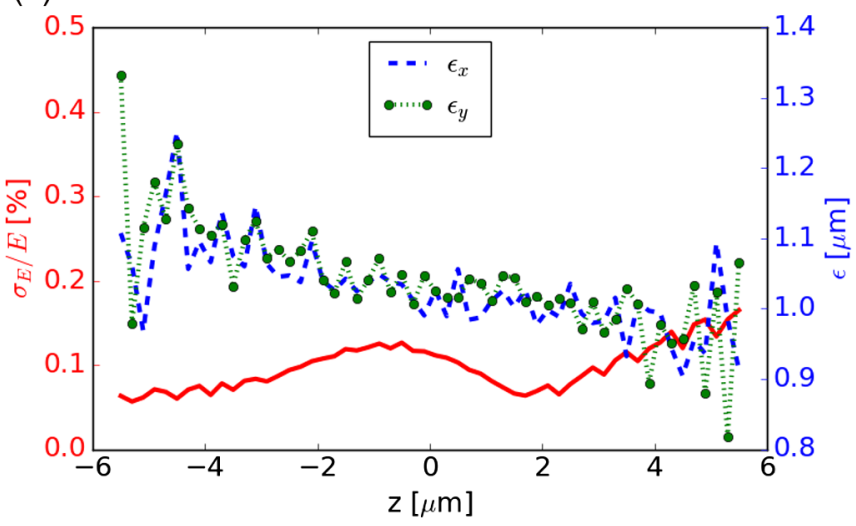

(b)

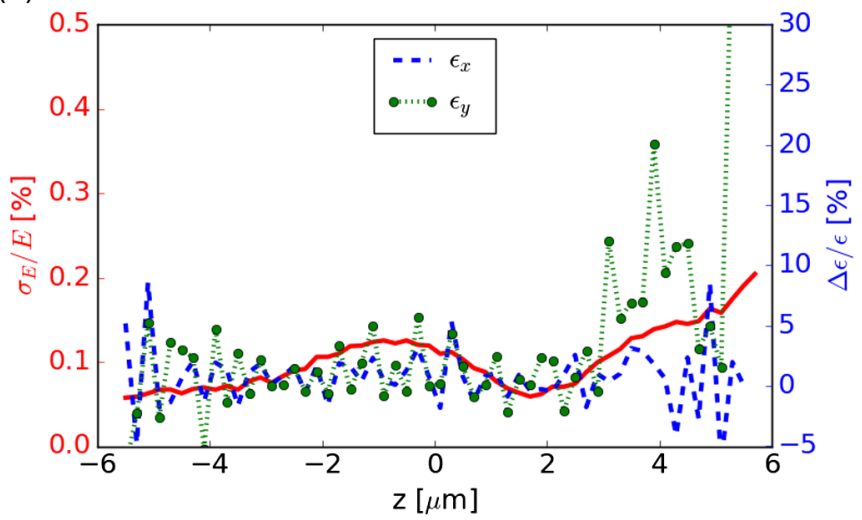

FIG. 10. Initial (a) and final (b) energy spread (red solid line) and horizontal (vertical) normalized slice emittance [blue (green) dashed line] in each $0.2 \mu \mathrm{m}$ long slice. 
In case there is a need to lower these gradients, there is still room to lengthen those magnets. With this line, the emittance increase is only $10 \%$ if the beam energy included in $\pm 1 \%$ of the nominal energy, that is, $84 \%$ of the total beam, is considered.

Another important parameter for the FEL is the slice emittance, that is, emittance of a given longitudinal slice. Its estimation tightly depends on the slice length. What matters for the FEL is the coherent length, which is $4.6 \mathrm{~nm}$ at the energy of $5 \mathrm{GeV}$. In our case, the shortest length that can be considered is around $0.2 \mu \mathrm{m}$ to get enough macroparticles for statistic calculations. With this length, the final beam current and average energy are given in Fig. 9. The initial and final energy spread and emittances are given in Fig. 10. As we can see, the variation of the slice emittance is very low (a few percent) because of the reduced slice energy spread. Because of the small number of particles in each slice, the results shown are rather noisy. Using a shorter slice length will give an even smaller slice emittance and slice energy spread, but the result is even noisier.

\section{CONCLUSIONS}

The principle of particle acceleration by a plasma-based setup is already well established. Its efficiency and feasibility have been demonstrated theoretically and experimentally. In the next steps that consist in sending the beam to either the users or to the next acceleration stage, beam matching out and matching in an acceleration stage along with the transfer line design are the key points, where preserving beam quality, especially its emittance, is the top issue. In this article, the phase and trace emittance change through a transfer line have been derived, pointing out the primary parameters involved in this process. Particle tracking in typical focusing configurations allowed us to confirm those behaviors of the emittance. Then 3D PIC code simulations for a given acceleration scheme of the EuPRAXIA project proved that the density ramp at the plasma entrance and exit are mandatory for preserving emittance. Finally, the design of a specific transfer line demonstrated that it is possible to deliver a beam right at the FEL entrance with characteristics meeting its very tight requirements.

This performance has been achieved thanks to the application of the following rules: (a) The plasma density up ramp length and the initial beam divergence are tuned so as to match the incoming beam to the focusing structure of the plasma acceleration stage, i.e., so that, at its entrance, the beam presents a waist and its rms size is equal to the square root of the wakefield focusing gradient. (b) The density down ramp length is tuned so that the Twiss parameter $\gamma$ at the exit is as low as possible while emittance growth in the ramp itself is not significant and is not spoiled by the nonlinear beam loading effect. (c) The transfer line should include six quadrupoles, aiming at achieving the four required transverse rms parameters at the FEL and the lowest emittance growth in the two transverse directions, i.e., in the smoothest way.

As a consequence, for a relatively high charge beam up to $30 \mathrm{pC}$, the emittance growth in the up ramp is $\sim 4 \%$, during acceleration $\sim 3 \%$, in the down ramp $\sim 4 \%$, and in the transfer line $\sim 10 \%$. Therefore, the overall emittance growth throughout the different sections is $\sim 22 \%$ of that at the plasma entrance.

\section{ACKNOWLEDGMENTS}

This work has received funding from the European Union's Horizon 2020 research and innovation program under Grant Agreement No. 653782. For numerical simulations, the authors were granted access to the High Performance Computing resources of [Très Grand Centre de Calcul du CEA/Centre Informatique National de l'Enseignement Supérieur/Institut du Développement et des Ressources en Informatique Scientifique] under the allocation A3 made by Genci (Grand Équipement National de Calcul Intensif).

\section{APPENDIX A: TRACE EMITTANCE AND PHASE EMITTANCE}

Let us first precise some notations and some basic statistical rules.

In the following, for a given random variable $a$, its average, rms, and variance are noted, respectively, by $\bar{a}, \sigma_{a}$, and $\left\langle a^{2}\right\rangle$. They are linked by the relations

$$
\sigma_{a}^{2}=\left\langle a^{2}\right\rangle=\overline{(a-\bar{a})^{2}}=\overline{a^{2}}-\bar{a}^{2} .
$$

If $a$ is a centered distribution,

$$
\bar{a}=0 \quad \text { and hence } \quad\left\langle a^{2}\right\rangle=\overline{a^{2}} .
$$

For two random variables $a$ and $b$, the covariance $\sigma_{a b}$ is given by

$$
\sigma_{a b}=\langle a b\rangle=\overline{(a-\bar{a})(b-\bar{b})}=\overline{a b}-\bar{a} \bar{b} .
$$

If $a$ and $b$ are statistically independent,

$$
\overline{a b}=\bar{a} \bar{b} \quad \text { and thus } \quad \sigma_{a b}=\langle a b\rangle=0 .
$$

In all this article, in order to simplify the writing, we assume that the transverse distributions are centered and, thus,

$$
\bar{x}=\overline{p_{x}}=0 .
$$

The two emittances, Eqs. (2) and (8), can then be written as, according to Eqs. (1) and (A1)-(A3),

$$
\varepsilon_{\mathrm{tr}}^{2}=\overline{x^{2}} \overline{x^{\prime 2}}-\overline{x x^{\prime 2}}
$$


and

$$
\varepsilon_{\mathrm{ph}}^{2}=\overline{x^{2}} \overline{x^{\prime 2} p_{z}^{2}}-\overline{x x^{\prime} p_{z}^{2}}
$$

If transverse and longitudinal distributions are independent, which is generally the case, according to (A4),

$$
\varepsilon_{\mathrm{ph}}^{2}=\overline{x^{2}} \overline{x^{\prime 2}} \overline{p_{z}^{2}}-\overline{x x^{\prime 2}}{\overline{p_{z}}}^{2} .
$$

This can be written, using Eqs. (A1) and (4)-(6),

$$
\varepsilon_{\mathrm{ph}, n}^{2}=\varepsilon_{\mathrm{tr}, n}^{2}\left(\frac{\overline{p_{z}^{2}}}{p_{0}^{2}}+\alpha^{2} \frac{\sigma_{p}^{2}}{p_{0}^{2}}\right),
$$

calling $p_{0}$ the average of the $p_{z}$ distribution.

Developing $p_{z}$ around this average value

$$
p_{z}=p_{0}\left(1+\frac{d p}{p_{0}}\right)
$$

implies that

$$
\frac{\overline{d p}}{p_{0}}=0
$$

then to the first order in $d p / p_{0}$, one has

$$
\overline{p_{z}^{2}} \approx p_{0}^{2}
$$

When in addition $\alpha=0$, i.e., at a beam waist, where the beam changes from divergent to convergent and vice versa, the two normalized emittances are equal:

$$
\varepsilon_{\mathrm{ph}, n}=\varepsilon_{\mathrm{tr}, n} \quad \text { when } \alpha=0 .
$$

Notice that Eq. (A9) is no longer valid in the presence of strong focusing elements which introduce significant dependences between $x^{\prime}$ and $p_{z}$, but the property given by Eq. (A13) remains always true. Indeed, a quadrupole will induce a spread in $x^{\prime}$ increasing linearly with the position $x$ and the momentum deviation $d p$. The typical pattern of such a beam in the $\left(x, x^{\prime}\right)$ phase space is two symmetrical isosceles triangles joined by the upper corner at the center of the phase space. At a waist, this pattern is straightened up, and the last terms $\overline{x x \prime}$ and $\overline{x x^{\prime} p_{z}}$ in Eqs. (A6) and (A7) are null. In the case of a waist in a focusing element, the beam size is maximum, and the pattern is horizontal, where particles having $p_{0}+p_{z}$ and $p_{0}-p_{z}$ are distributed symmetrically in $+x$, and $-x^{\prime}$, so that $\overline{x^{\prime 2} p_{z}^{2}}=\overline{x^{\prime 2}} \overline{p_{z}^{2}}$. In the case of a waist in a free drift, the beam size $\overline{x^{2}}$ is minimum, close to zero, which, once multiplied by the constant $\overline{x^{\prime 2}}$ and $\overline{x^{\prime 2} p_{z}^{2}}$ in the drift, makes the difference between the two emittances minimum, so that they are equal around this waist. We have also checked the validity of Eq. (A13) with simulations in many different configurations and carefully tracked each term of the expression of the two emittances to understand the mechanism leading to this property.

\section{APPENDIX B: EMITTANCE EVOLUTION THROUGH A FREE DRIFT}

Through a drift of length $l$, the coordinates change as

$$
x=x_{0}+x_{0}^{\prime} l, \quad x^{\prime}=x_{0}^{\prime},
$$

where the subscript 0 corresponds to the drift entrance and no subscript corresponds to the drift exit. With this, the trace emittance [Eq. (A6)] at the drift exit can be expressed exclusively in terms of coordinates at the drift entrance:

$$
\varepsilon_{\mathrm{tr}}^{2}=\left(\overline{x_{0}^{2}}+2 l \overline{x_{0} x_{0}^{\prime}}+\overline{x_{0}^{\prime 2}} l^{2}\right) \overline{x_{0}^{\prime 2}}-\left(\overline{x_{0} x_{0}^{\prime}}+\overline{x_{0}^{\prime 2}} l\right)^{2},
$$

hence we can see that the trace emittance remains constant in a free drift:

$$
\varepsilon_{\mathrm{tr}}^{2}-\varepsilon_{\mathrm{tr} 0}^{2}=0
$$

The variation of the phase emittance can be calculated in the same way from Eq. (A7) using Eq. (1):

$$
\begin{aligned}
\varepsilon_{\mathrm{ph}}^{2}-\varepsilon_{\mathrm{ph} 0}^{2}= & 2 l\left(\overline{x_{0} x_{0}^{\prime}} \overline{x_{0}^{\prime 2} p_{z}^{2}}-\overline{x_{0} x_{0}^{\prime} p_{z}} \overline{x_{0}^{\prime 2} p_{z}}\right) \\
& +l^{2}\left[\overline{x_{0}^{\prime 2}} \overline{x_{0}^{\prime 2} p_{z}^{2}}-\left(\overline{x_{0}^{\prime 2} p_{z}}\right)^{2}\right] .
\end{aligned}
$$

If transverse and longitudinal distributions are statistically independent, and by expressing in terms of the initial Twiss parameters given by Eqs. (4)-(6), one can obtain

$$
\varepsilon_{\mathrm{ph}, n}^{2}-\varepsilon_{\mathrm{ph} 0, n}^{2}=\varepsilon_{\mathrm{tr} 0, n}^{2}\left(\frac{\sigma_{p}}{p_{0}}\right)^{2} \gamma_{0} l\left(\gamma_{0} l-2 \alpha_{0}\right) .
$$

\section{APPENDIX C: EMITTANCE EVOLUTION THROUGH A THIN LENS}

Through a thin lens of integrated normalized gradient $k=K L$, the coordinates change as

$$
x=x_{0}, \quad x^{\prime}=k x_{0}+x_{0}^{\prime},
$$

where the subscript 0 corresponds now to the lens entrance and no subscript corresponds to the lens exit. Notice that the focusing gradient experienced by a particle is inversely proportional to its momentum. Based on the expression of $p_{z}$ in Eq. (A10), $k$ can be developed as

$$
k=k_{0}\left[1-\frac{d p}{p_{0}}+\frac{1}{2}\left(\frac{d p}{p_{0}}\right)^{2}\right],
$$


where $k_{0}$ is the integrated normalized gradient seen by the particle with nominal energy. $k$ is thus a distribution linked to the longitudinal motion, for which the averages $\bar{k}$ and $\overline{k^{2}}$ can be calculated as functions of $\frac{d p}{p_{0}}$. As it is a centered distribution, averages of its odd powers vanish, and, neglecting its fourth order, we have

$$
\overline{k^{2}}-\bar{k}^{2}=k_{0}^{2}\left(\frac{\sigma_{p}}{p_{0}}\right)^{2} \text {. }
$$

The trace emittance after lens crossing given in Eq. (A6) can be expressed exclusively with coordinates at the lens entrance:

$$
\varepsilon_{\mathrm{tr}}^{2}=\overline{x_{0}^{2}}\left(\overline{k^{2} x_{0}^{2}}+2 \overline{k x_{0} x_{0}^{\prime}}+\overline{x_{0}^{\prime 2}}\right)-\left(\overline{k x_{0}^{2}}+\overline{x_{0} x_{0}^{\prime}}\right)^{2},
$$

hence

$$
\varepsilon_{\mathrm{tr}}^{2}-\varepsilon_{\mathrm{tr} 0}^{2}=2\left(\overline{k x_{0} x_{0}^{\prime}} \overline{x_{0}^{2}}-\overline{k x_{0}^{2}} \overline{x_{0} x_{0}^{\prime}}\right)+\overline{k^{2} x_{0}^{2}} \overline{x_{0}^{2}}-{\overline{k x_{0}^{2}}}^{2} .
$$

If transverse and longitudinal distributions are independent, we can obtain using Eqs. (4) and (C3)

$$
\frac{\varepsilon_{\mathrm{tr}}^{2}-\varepsilon_{\mathrm{tr} 0}^{2}}{\varepsilon_{\mathrm{tr} 0}^{2}}=\beta_{0}^{2} k^{2}\left(\frac{\sigma_{p}}{p_{0}}\right)^{2} .
$$

Let us switch to the calculation of the phase emittance as given in Eq. (A7) at the lens exit. It can be expressed as a function of coordinates at the lens entrance:

$$
\begin{aligned}
\varepsilon_{\mathrm{ph}}^{2}= & \overline{x_{0}^{2}}\left(\overline{k^{2} x_{0}^{2} p_{z}^{2}}+2 \overline{k x_{0} p_{x_{0}} p_{z}}+\overline{{p_{x_{0}}}^{2}}\right) \\
& -\left(\overline{k x_{0}^{2} p_{z}}+\overline{x_{0} p_{x_{0}}}\right)^{2},
\end{aligned}
$$

and the variation through the thin lens is

$$
\begin{aligned}
\varepsilon_{\mathrm{ph}}^{2}-\varepsilon_{\mathrm{ph} 0}^{2}= & 2\left(\overline{k x_{0} p_{x_{0}} p_{z}} \overline{x_{0}^{2}}-\overline{k x_{0}^{2} p_{z}} \overline{x_{0} p_{x_{0}}}\right) \\
& +\overline{k^{2} x_{0}^{2} p_{z}^{2}} \overline{x_{0}^{2}}-\overline{k x_{0}^{2} p_{z}^{2}} .
\end{aligned}
$$

If transverse and longitudinal distributions are independent, the phase emittance remains constant when crossing a thin lens:

$$
\varepsilon_{\mathrm{ph}}^{2}-\varepsilon_{\mathrm{ph} 0}^{2}={\overline{x_{0}^{2}}}^{2}\left(\overline{k^{2} p_{z}^{2}}-{\overline{k p_{z}}}^{2}\right)=0 .
$$

This section clearly shows the different behaviors of the two emittances when crossing a thin lens: the trace emittance is subjected to a jump proportional to the square of the focusing strength, while the phase emittance remains unchanged. Although the thin lens induces a coupling between transverse and longitudinal motion via the $p_{z}$ depending on focusing strength $k$ [Eqs. (C1) and (C3)], separating $x$ and $z$ dependencies is still possible, allowing one to analytically calculate the precise behavior of the emittances.
[1] T. Tajima and J. M. Dawson, Laser Electron Accelerator, Phys. Rev. Lett. 43, 267 (1979).

[2] J. Faure, Y. Glinec, A. Pukhov, S. Kiselev, S. Gordienko, E. Lefebvre, J.-P. Rousseau, F. Burgy, and V. Malka, A laser-plasma accelerator producing monoenergetic electron beams, Nature (London) 431, 541 (2004).

[3] E. Esarey, C. B. Schroeder, and W. P. Leemans, Physics of laser-driven plasma-based electron accelerators, Rev. Mod. Phys. 81, 1229 (2009).

[4] M. Litos et al., High-efficiency acceleration of an electron beam in a plasma wakefield accelerator, Nature (London) 515, 92 (2014).

[5] W. P. Leemans, A. J. Gonsalves, H.-S. Mao, K. Nakamura, C. Benedetti, C. B. Schroeder, Cs. Tóth, J. Daniels, D. E. Mittelberger, S. S. Bulanov, J.-L. Vay, C. G. R. Geddes, and E. Esarey, Multi-GeV Electron Beams from CapillaryDischarge-Guided Subpetawatt Laser Pulses in the SelfTrapping Regime, Phys. Rev. Lett. 113, 245002 (2014).

[6] S. Cipiccia et al., A tuneable ultra-compact high-power, ultra-short pulsed, bright gamma-ray source based on bremsstrahlung radiation from laser-plasma accelerated electrons, J. Appl. Phys. 111, 063302 (2012).

[7] K. Khrennikov, J. Wenz, A. Buck, J. Xu, M. Heigoldt, L. Veisz, and S. Karsch, Tunable All-Optical Quasimonochromatic Thomson X-Ray Source in the Nonlinear Regime, Phys. Rev. Lett. 114, 195003 (2015).

[8] V. Malka, J. Faure, Y. A. Gauduel, E. Lefebvre, A. Rousse, and K. T. Phuoc, Principles and applications of compact laser-plasma accelerators, Nat. Phys. 4, 447 (2008).

[9] M. E. Couprie, A. Loulergue, M. Labat, R. Lehe, and V. Malka, Towards a free electron laser based on laser plasma accelerators, J. Phys. B 47, 234001 (2014).

[10] P. A. Walker et al., Horizon 2020 EuPRAXIA design study, J. Phys. Conf. Ser. 874, 012029 (2017).

[11] S. Steinke, J. van Tilborg, C. Benedetti, C. G. R. Geddes, C. B. Schroeder, J. Daniels, K. K. Swanson, A. J. Gonsalves, K. Nakamura, N. H. Matlis, B. H. Shaw, E. Esarey, and W. P. Leemans, Multistage coupling of independent laser-plasma accelerators, Nature (London) 530, 190 (2016).

[12] J. Luo, M. Chen, W. Y. Wu, S. M. Weng, Z. M. Sheng, C. B. Schroeder, D. A. Jaroszynski, E. Esarey, W. P. Leemans, W. B. Mori, and J. Zhang, Multistage Coupling of LaserWakefield Accelerators with Curved Plasma Channels, Phys. Rev. Lett. 120, 154801 (2018).

[13] T. Mehrling, J. Grebenyuk, F. S. Tsung, K. Floettmann, and J. Osterhoff, Transverse emittance growth in staged laserwakefield acceleration, Phys. Rev. ST Accel. Beams 15, 111303 (2012).

[14] K. Floettmann, Adiabatic matching section for plasma accelerated beams, Phys. Rev. ST Accel. Beams 17, 054402 (2014).

[15] I. Dornmair, K. Floettmann, and A. R. Maier, Emittance conservation by tailored focusing profiles in a plasma accelerator, Phys. Rev. ST Accel. Beams 18, 041302 (2015).

[16] P. Lee, G. Maynard, T. L. Audet, B. Cros, R. Lehe, and J.-L. Vay, Optimization of laser-plasma injector via beam loading effects using ionization-induced injection, Phys. Rev. Accel. Beams 21, 052802 (2018). 
[17] M. Migliorati, A. Bacci, C. Benedetti, E. Chiadroni, M. Ferrario, A. Mostacci, L. Palumbo, A. R. Rossi, L. Serafini, and P. Antici, Intrinsic normalized emittance growth in laser-driven electron accelerators, Phys. Rev. ST Accel. Beams 16, 011302 (2013).

[18] M. Scisciò, L. Lancia, M. Migliorati, A. Mostacci, L. Palumbo, Y. Papaphilippou, and P. Antici, Parametric study of transport beam lines for electron beams accelerated by laser-plasma interaction, J. Appl. Phys. 119, 094905 (2016).

[19] A. Loulergue, M. Labat, C. Evain, C. Benabderrahmane, V. Malka, and M. E. Couprie, Beam manipulation for compact laser wakefield accelerator based free-electron lasers, New J. Phys. 17, 023028 (2015).

[20] M. Rossetti Conti, A. Bacci, A. Giribono, V. Petrillo, A. R. Rossi, L. Serafini, and C. Vaccarezza, Electron beam transfer line design for plasma driven Free Electron Lasers, Nucl. Instrum. Methods Phys. Res., Sect. A 909, 84 (2018).

[21] S. Fartoukh, Ph.D. thesis, Méthodes d'analyse d'une ligne de focalisation finale dans le cadre du projet du collisionneur linéaire TESLA, Reports No. CERN-Thesis2000-035, No. DAPNIA-SEA-98-02-T, and No. CMP00078603, Eq. (3,15), p. 118, 1997.
[22] D. Uriot and N. Pichoff, in Proceedings of the International Particle Accelerator Conference, Richmond, VA, USA (JACoW, Geneva, Switzerland, 2015), MOPWA008.

[23] X. Li, A. Mosnier, and P. A. P. Nghiem, Design of a $5 \mathrm{GeV}$ laser-plasma accelerating module in the quasi-linear regime, Nucl. Instrum. Methods Phys. Res., Sect. A 909, 49 (2018).

[24] X. Li, P. A. P. Nghiem, and A. Mosnier, Toward low energy spread in plasma accelerators in quasilinear regime, Phys. Rev. Accel. Beams 21, 111301 (2018).

[25] K. Floettmann, http://www.desy.de/ mpyflo.

[26] J.-L. Vay, C. G. R. Geddes, E. Esarey, C. B. Schroeder, W. P. Leemans, E. Cormier-Michel, and D. P. Grote, Modeling of $10 \mathrm{GeV}-1 \mathrm{TeV}$ laser-plasma accelerators using Lorentz boosted simulations, Phys. Plasmas 18, 123103 (2011).

[27] T. L. Audet, P. Lee, G. Maynard, S. Dobosz Dufrénoy, A. Maitrallain, M. Bougeard, P. Monot, and B. Cros, Gas cell density characterization for laser wakefield acceleration, Nucl. Instrum. Methods Phys. Res., Sect. A 909, 383 (2018).

[28] J. Kennedy and R. Eberhart, in Proceedings of the IEEE International Conference on Neural Networks, Perth, WA, Australia (IEEE, 1995). 